\title{
Isoflurane Provides Neuroprotection in Neonatal Hypoxic Ischemic Brain Injury
}

\author{
Sherrefa R Burchell, BS ${ }^{1}$, Brandon J Dixon, BS ${ }^{1}$, Jiping Tang, MD ${ }^{1}$, and John H Zhang, MD, \\ $\mathrm{PhD}^{1,2,{ }^{*}}$ \\ ${ }^{1}$ Department of Physiology and Pharmacology, Loma Linda University School of Medicine, Risley \\ Hall, Room 223, Loma Linda, CA 92354, USA \\ ${ }^{2}$ Department of Neurosurgery, Loma Linda University School of Medicine, Loma Linda, CA, USA
}

\section{Abstract}

Isoflurane is a volatile anesthetic that is widely used clinically as an inhalational anesthetic. In recent years, several studies have indicated that isoflurane has neuroprotective properties. This has led to the beneficial effects of isoflurane being analyzed in both cell culture and animal models, including various models of brain injury. Neonatal hypoxia ischemia may be characterized as injury that occurs in the immature brain, resulting in delayed cell death via excitotoxicity and oxidative stress. These adverse events in the developing brain often lead to detrimental neurological defects in the future. Currently, there are no well-established effective therapies for neonatal hypoxia ischemia. In line with this, isoflurane, which displays neuroprotective properties in several paradigms and has been shown to improve neurological deficits caused by brain injuries, has the capability to be an extremely relevant clinical therapy for the resolution of deficits concomitant with neonatal hypoxic ischemic brain injuries. This review will therefore seek to explore and analyze the current information on isoflurane, looking at general isoflurane anesthetic properties, and the protection it confers in different animal models, focusing particularly on neuroprotection as shown in studies with neonatal hypoxic ischemic brain injury.

\section{Keywords}

Isoflurane; Long-term neuroprotection; Pre-conditioning; Post-conditioning; Isoflurane mechanism; Neonatal hypoxia ischemia

\section{Isoflurane and Other Volatile Anesthetics}

Isoflurane is a volatile anesthetic that has been in clinical practice for several decades. Volatile anesthetics are also known as inhalational anesthetics and, apart from isoflurane, also include sevoflurane, desflurane, halothane, and enflurane. Of the five major volatile anesthetics, isoflurane, sevoflurane, and desflurane are most commonly used today. Halothane and enflurane are not used widely due to undesirable side effects ${ }^{1}$. The volatile anesthetics all differ in potency, adverse effects, and cost, and are used extensively during surgery in human neonates and during neonatal animal research. Moreover, volatile

*Corresponding author. Department of Neurosurgery, Loma Linda University School of Medicine, Loma Linda, CA, USA, Tel: 909-558-4723, Fax: 909-558-0119, johnzhang3910@yahoo.com.

Publisher's Disclaimer: This is a PDF file of an unedited manuscript that has been accepted for publication. As a service to our customers we are providing this early version of the manuscript. The manuscript will undergo copyediting, typesetting, and review of the resulting proof before it is published in its final citable form. Please note that during the production process errors may be discovered which could affect the content, and all legal disclaimers that apply to the journal pertain. 
anesthetics are employed in about $80 \%$ of the surgeries in the United States that are performed under general anesthesia ${ }^{2}$. Volatile anesthetics have been reported to produce their effects by a number of mechanisms, including antagonism of ionic channels, activation of glycine and $\gamma$-aminobutyric acid (GABA) receptors, and alteration of the function and activity of other cellular proteins, as well as through utilizing high lipid solubility characteristics ${ }^{3,4}$.

Isoflurane (2-chloro-2-(difluoromethoxy)-1,1,1-trifluoro-ethane, $\mathrm{CHF}_{2}-\mathrm{O}-\mathrm{CHCl}-\mathrm{CF}_{3}$ ), has been shown to be more potent than several other anesthetics. It is much more widely used in comparison to the other inhalational fluorinated inhalational anesthetics ${ }^{5}$. Isoflurane was discovered in 1965 by Dr. Ross Terrell and associates in their quest for a better inhaled anesthetic than was available at the time and was the $469^{\text {th }}$ compound found, following enflurane and halothane ${ }^{5}$. Isoflurane has a minimum alveolar concentration (MAC) of $1.15 \%$, and blood/gas partition coefficient of 1.4, lower than that of most other inhaled anesthetics ${ }^{6}$. Also, isoflurane is very stable and is resistant to biodegradation ${ }^{3}$. These properties of isoflurane allow it to rapidly induce unconsciousness and also to be quickly eliminated from circulation.

\section{Isoflurane is Protective in Various Animal Models}

Isoflurane has been studied in animal models of various diseased states, such as Lipopolysaccharide (LPS)-induced Acute Inflammation of the Lung ${ }^{7}$, Acute Lung Injury ${ }^{8}$, glucose-induced oxidative stress ${ }^{9}$, renal ischemia/reperfusion injury ${ }^{10,11}$, cardiac injury ${ }^{12}$, etc. In these models, it is shown to provide protection from injury and improve various negative functional outcomes. The protective effects of isoflurane in these models are reported to occur through the activation of several pathways, and are briefly outlined in Table 1.

In particular, isoflurane shows neuroprotection in several stroke models including subarachnoid hemorrhage $(\mathrm{SAH})^{13}$, middle cerebral artery occlusion $(\mathrm{MCAO})^{14,15}$, intracerebral hemorrhage ${ }^{16}$, traumatic brain injury ${ }^{17}$, and Neonatal Hypoxic Ischemic Brain Injury (HI) ${ }^{18}$ (Table 1). For this review, the main focus will be on the neuroprotection of isoflurane as studied in neonatal HI, its possible mechanisms of action, and what direction this could be leading us in terms of clinical applications of isoflurane treatment.

\section{Neonatal Hypoxia-Ischemia}

Neonatal Hypoxia Ischemia is a major cause of mortality and neurological deficits such as cerebral palsy, mental retardation, and epilepsy in the perinatal period ${ }^{19,20}$. Several factors have been implicated in the pathophysiology of HI, including inflammatory mediators, excitotoxicity, and oxidative stress ${ }^{21}$. While extensive research has been done on this form of brain injury, there is a gap between the basic and clinical sciences as successful treatments are still lacking. There is still a significant number of neonates who suffer from perinatal $\mathrm{HI}$ and its repercussions, approximately 1 in 4000, a figure two to three times higher than the incidence of childhood stroke ${ }^{22}$. The methods of interventions are often limited for neonatal HI, since neuronal cell death is frequently only delayed, although it usually appears to be prevented shortly after an assault. Thus, it is essential that more preclinical studies be carried out to elucidate the possibility of an effective therapy that will provide long-term neuroprotection against $\mathrm{HI}$ in the perinatal period, reducing the effects seen up to adulthood.

To study neonatal hypoxic ischemic brain injury, the Rice-Vannucci model is the one that is most widely used. Induction is most commonly with isoflurane at $3 \%$ and occurs for 4 minutes, followed by ligation of the right common carotid artery in rat pups. Isoflurane is 
continuously infused for the duration of the surgery (typically not longer than 5 minutes) at $2.5 \%$. The inspired concentration of isoflurane is lowered (from $3 \%$ to $2.5 \%$ ) as this compensates for the decreased uptake due to the equilibration of isoflurane in the body following the induction period ${ }^{5}$. The pups are then subjected to a hypoxic chamber with $8 \%$ oxygen at $37^{\circ} \mathrm{C}$ for 2.5 hours. Infarction volumes are calculated and other neurological assessments are done at different time points after surgery. This method has been modified from that described by Rice, Vannucci, and Brierly, and is most commonly done in postnatal day 7 or 10 rats $^{23}$. P7 rat pups represent a human fetus at gestational age 32 to 34 weeks or a newborn infant at this stage ${ }^{24}$, while P10 rats are similar to the older neonate approaching adolescent stages of development or a full-term neonate ${ }^{25}$.

\section{Neuroprotection of Isoflurane in Neonatal HI: Possible Mechanisms}

The use of isoflurane as an anesthetic in the surgery for modeling neonatal HI, along with the great variations observed in infarct sizes within and among litters, particularly as surgery times varied has led to speculations on the effects that isoflurane may be having on these neonates. This, among other reasons, has therefore sparked the interest in studying whether or not isoflurane may be providing some form of neuroprotection against ischemic injury in neonatal rat pups.

As a result, several studies have found that isoflurane, when it is administered before or after an experimental hypoxic-ischemic insult (pre- and post-conditioning) in neonates, has the ability to reduce negative outcomes. Studies indicate that isoflurane acts through a number of mechanisms that are involved with neuroprotection and an increase in neuronal viability (Figure 1). It has been suggested that isoflurane inhibits excitotoxicity that is initiated by an accumulation of glutamate during ischemia, resulting in a reduction in necrosis. The antagonism of glutamate receptors by isoflurane has been shown in in vitro studies, as well as in in vivo models where isoflurane reduced extracellular glutamate during ischemia ${ }^{26}$. In addition, isoflurane has been shown to be a GABA receptor agonist, providing an inhibitory effect against excitotoxicity ${ }^{3}$.

Under hypoxic conditions isoflurane was shown to be protective via its interaction with the pathways that involve phospholipase $\mathrm{C}$ and the release of $\mathrm{Ca}^{2+}$ from intracellular stores. Phospholipase C triggers the Phosphatidylinositol-3-kinase/Protein Kinase B and Mitogenactivated protein kinase (PI3K/Akt/MAPK) pathways, which are anti-apoptotic ${ }^{27}$. Isoflurane also induced neuroprotection by altering the phosphorylation states of players in both the calcium-dependent and calcium-independent MAP kinase pathways, increasing the phosphorylation of some of the kinases (Pyk-2, ERK, MKK-6, JNK), while decreasing the phosphorylation of the downstream MAP kinase dependent transcription factors (p38, pElk-1 p90 RSK, ATF-2). Phosphorylation of the MAP kinases was prevented by an inositol triphosphate (IP3) receptor antagonist, suggesting that this phenomenon after isoflurane preconditioning depends on the small increases in intracellular $\mathrm{Ca}^{2+}$. The study also showed that isoflurane enabled neurons to avoid potentially toxic levels of $\mathrm{Ca}^{2+}$ increase. Moreover, there was an observed increase in the levels of Akt, an anti-apoptotic protein, due to isoflurane preconditioning ${ }^{27,28}$. The activation of the Akt pathway was also observed in a model of oxidative and inflammatory stress on cardiac myocytes. Isoflurane was shown to prevent apoptosis by activating Akt and enhancing B-cell lymphoma-2 (Bcl-2) expression ${ }^{29,30}$.

Furthermore, isoflurane was shown to protect against oxygen glucose deprivation-induced ischemia in neuronal cultures by activating the cell-surviving protein, hypoxia inducible factor -1a (HIF-1a) and the subsequent increase in expression of inducible nitric oxide synthase (iNOS) mRNA. Notably, the protection observed with isoflurane preconditioning 
was also dependent on the extracellular signal-related kinase (ERK) pathway; an ERK1/2 inhibitor partially reversed the protective effects that resulted from isoflurane administration ${ }^{31}$. The aforementioned studies were mainly carried out in in vitro models of hypoxia, and though they may provide clearer mechanistic pathway descriptions, they differ from the physiology seen in intact animals. However, similar neuroprotection has been observed in vivo.

In one study done in an animal model of neonatal HI, Chen et.al, hypothesized that longer isoflurane exposure times may be one of the underlying factors leading to the inconsistent results in infarction volumes observed in the model. The results showed that longer surgery times, and hence longer isoflurane exposure, resulted in decreased infarction severity in the rat pups. Pups that had exposure times to isoflurane of 13 or 21 minutes, had significantly less volume of infarction than those with only 5 minutes of exposure: $33.2 \%$ in the 5 minute group, versus $20.3 \%$ in the 13 minute group and $11.3 \%$ in the 21 minute group in the 7-day old pups. This trend was also observed in the 10-day old rat pups (24.8\% in the 5-minute group versus $11.9 \%$ in the 13 -minute group and $9.3 \%$ in the 21 -minute group $)^{25}$.

By extension, this group also did experiments to elucidate the mechanism by which isoflurane was participating in this neuroprotective role. They found that the sphingosine-1kinase/PI3K/Akt (S1P/PI3K/Akt) pathway is active in this function as administration of selective inhibitors of S1P (VPC23019) and PI3K (Wortmannin) completely reversed the effects of isoflurane-induced neuroprotection. Importantly, isoflurane provided both short and long-term neuroprotection as effects were observed up to 4 weeks after the HI injury, when neurobehavioral tests were carried out. The effect of the S1P and PI3K inhibitors had a long-term effect as well, reversing the improvement in neurobehavior induced by longer exposures to isoflurane. Also, Akt phosphorylation was preserved in the isoflurane pretreated group, and a significant reduction in cleaved caspase-3 levels was observed. These findings confirmed the speculation of the involvement of the S1P/PI3K/Akt pathway ${ }^{18}$. In addition, in a SAH model, isoflurane was also shown to be neuroprotective by activating the sphingosine kinase/S1P (SK/S1P) pathway. Isoflurane treatment attenuated the disruption of the blood-brain-barrier (BBB) that usually occurs after $\mathrm{SAH}^{13}$.

The involvement of the S1P/PI3K/Akt pathway in protection has also been implicated by other groups in different models of ischemia. Isoflurane was found to protect against renal dysfunction, significantly lowering plasma creatinine values and tubular necrosis, an effect abrogated by SK and S1P receptor antagonists, providing further evidence that their activity is essential to isoflurane-induced protection against ischemia. Additionally, mice lacking the SK1 enzyme were not protected against injury from ischemia ${ }^{10}$. The S1P pathway involvement in protection was seen in cardiac ischemia reperfusion injury also as the administration of S1P, which activated the S1P receptor, resulted in reduced infarct sizes, leukocyte recruitment and adhesion ${ }^{32}$. In models of liver ischemia, the SK/SIP pathway was also shown to be activated. There was a significant improvement in hepatic function in both normal and cirrhotic livers, increased anti-inflammatory cytokine production, and downregulation of anti-apoptotic genes following ischemia-reperfusion injury due to the activation of $\mathrm{S}_{1} \mathrm{P}^{33}$.

Volatile anesthetics, including isoflurane, have been proposed to be exhibit potency as a result of their high lipid solubility ${ }^{3}$. This theory was proposed by Meyer and Overton in 1901 and has been used to explain the involvement of the lysophospholipid, Sphingosine-1phosphate, which results from the activation of sphingomyelin hydrolysis ${ }^{34}$. Sphingomyelin is a lipid component of the membrane which is hydrolyzed to ceramide, a precursor of sphingosine. Sphingosine is then converted to S1P by the enzyme sphingosine kinase ${ }^{10}$. Spingosine kinase is involved in many different biological processes including cell growth 
and cell survival ${ }^{35}$. In addition, sphingosine kinase and S1P have been shown to upregulate the ERK/MAPK pathway, resulting in increased cell survival ${ }^{36,37}$. A significant finding was that animals with a knockout of the SK gene could not be protected by isoflurane treatment ${ }^{10,38}$. These studies all correlate with the Lipid Theory which describes the potency of volatile anesthetics in relation to lipid solubility. However, in recent years, the Protein Theory, which postulates that anesthetics act directly on proteins rather than accumulating in membranes, has stimulated a lot of interest ${ }^{3,39}$.

Consequently, other mechanisms have been proposed on how isoflurane confers neuroprotection. One such pathway is through the activation of inducible nitric oxide synthases (iNOS). Zhao, et.al found that isoflurane induced an increase in nitric oxide and suggested that this may be the mediator for the neuroprotection induced by isoflurane. They confirmed this by the use of a nitric oxide inhibitor, aminoguanidine, which abolished the neuroprotection conferred by isoflurane ${ }^{22}$. Isoflurane neuroprotection was shown using a preconditioning model where isoflurane was given at $1.5 \%$ for 30 minutes 24 hours before the hypoxic insult. Neuroprotection was measured up to 7 days post injury.

In another study by the same group, it was found that the brain loss induced by HI was significantly attenuated up to one month following injury, when animals were preconditioned with $1.5 \%$ isoflurane for 30 minutes 24 hours before injury. This conclusion was made as there were no significant differences between control and isoflurane treated groups in long-term reference memory, as well as short-term working memory. Isoflurane also significantly improved motor coordination one month after injury. Additionally, preconditioning with isoflurane resulted in increased Bcl-2 expression in the hippocampus ${ }^{40}$. Bcl-2 can decrease the release of cytochrome $\mathrm{C}$ and mitochondrial membrane permeability caused by ischemic episodes ${ }^{40}$. Again, it was found that the protection by isoflurane could be reversed by administration of an inducible nitric oxide synthase inhibitor, suggesting a role for iNOS in the isoflurane-induced neuroprotection. Small increases in iNOS protein expression have been associated with cardioprotection and neuroprotection. iNOS is believed to be an important factor for protection since protein kinase C(PKC) and MAP kinase are both located downstream of iNOS and are believed to provide protection ${ }^{41}$. Notably, isoflurane was found to increase the p38 MAPK phosphorylation status after an ischemic injury and the reduction induced by preconditioning with isoflurane was abolished by the administration of a p38 MAPK inhibitor ${ }^{42}$.

Kapinya et.al. also found that isoflurane preconditioning resulted in an increase in iNOS in an MCAO model and suggested that the increase in iNOS may be the means by which isoflurane reduces infarction volume and other neurological deficits. However, they also stated that further study is needed to identify the underlying anesthetic mechanism as the increase in iNOS was not observed until 6 hours after the preconditioning event, while protection was seen immediately after preconditioning with isoflurane ${ }^{43}$. Nitric oxide is known to interact with two survival pathways: the Ras/Raf/MEK/ERK pathway and the PI3K/Akt pathway ${ }^{44}$. Both of these pathways are essential to neuronal survival and could therefore be the underlying mechanism by which the isoflurane-induced increase in iNOS is protective in brain injury models. However, there is some contradiction in this area as NO has been shown to be both protective and toxic ${ }^{44}$. Therefore, there are still further studies that need to be done to clarify the neuroprotection of isoflurane via an increase in iNOS.

The studies described above show a long-term neuroprotective role of the anesthetic, isoflurane. This is significant as a large percentage of infants who suffer from neonatal HI do survive into adulthood; however, most of them have residual motor and cognitive deficits ${ }^{21}$. However, importantly, there have been contradictions on whether or not the neuroprotection provided by isoflurane is long-term, or only transient. A study done by 
McAuliffe, et.al, suggested that isoflurane may be useful in preventing the deficits seen long after the neonatal injury, or at least reducing the severity. This conclusion was made from experiments that showed that preconditioning with isoflurane improved striatal function in adulthood. Neurobehavioral testing was also done by the use of a water maze with adult mice that had undergone neonatal HI, and was done at 70 days old. Mice preconditioned with isoflurane performed as well as sham in this neurobehavioral test and this therefore provides evidence for long-term neuroprotection ${ }^{45}$.

By contrast, Sasaoka, et.al found that preconditioning with isoflurane at $2 \%$ for either 60 or 90 minutes before the $\mathrm{HI}$ insult induced ischemic tolerance in the hippocampus of P7 rat pups up to 7 days after the injury, but not at 49 days $^{20}$. These results suggest that the neuroprotective effect of isoflurane is only transient. This may be due to the rapid removal of isoflurane from the system as a result of small amounts of biodegradation, as stated before.

It has also been suggested that isoflurane preconditioning may simply be delaying neuronal injury, not preventing it. This idea was proposed based on results that showed that brain infarction in the isoflurane treated group was significantly reduced in comparison to controls two days following ischemic injury, but this reduction was not seen 14 days after the insult. However, isoflurane did reduce the number of necrotic cells in the penumbral area two weeks after ischemia was induced ${ }^{46}$. This experiment was carried out in an adult model of ischemia. Nevertheless, it still presents a significant consideration to be made with regards to the use of isoflurane for neuroprotection clinically because the delay in neuronal injury provided by isoflurane may provide a longer therapeutic window for other interventions.

\section{Future Directions}

It is clear that isoflurane exhibits neuroprotective properties, and harnessing this capability of the volatile anesthetic may be very relevant clinically. However, while isoflurane has been shown to be beneficial in numerous cases, before it can be used as a neuroprotective therapy for neonatal HI, several concerns have to be addressed. One such consideration is the length of time that isoflurane is administered. Studies have shown that isoflurane exposure for periods of 3 to 6 hours resulted in caspase activation and neuronal death ${ }^{47,48}$, in contrast to the potent neuroprotection observed with shorter exposure times ${ }^{25,40}$. Therefore, studies specifically aimed at establishing an optimal time period within which there is still effective neuroprotection from isoflurane, without causing neurotoxicity, have to be done.

There are contradictory evidences in support of isoflurane playing a neuroprotective role, while at the same time, potentially being neurotoxic. As discussed above, there are many animal studies that show isoflurane to be convincingly neuroprotective. However, evidence based on clinical studies in humans is not as strong ${ }^{4}$. And while there is not much evidence on the neurotoxicity resulting from isoflurane anesthesia, the fact that there is any, is reason for considerations to be made in this regard before clinical isoflurane therapy can progress substantially.

Isoflurane studies are clinically relevant and translatable because the percentage of isoflurane used experimentally is around $1.15 \%$ to $2 \%$, which is comparable to a MAC of $1.15 \%$ in human adults and $1.6 \%$ in human neonates ${ }^{40}$. However, different researchers have found the most effective dosages to be different in their models. With these discrepancies, much more would need to be done to establish what the optimal dosage of isoflurane is that will be mosy effective, providing maximal neuroprotection to infants.

Another factor that is very important to consider is elucidating the exact mechanism by which isoflurane provides protection from cell death and other negative outcomes. Several 
different pathways have been implicated to date. It was first postulated that isoflurane, and volatile anesthetics by extension, were more potent the more lipid-solubility they exhibited ${ }^{3}$. Since the membrane is composed mainly of lipids, they were thought to act through membrane lipids. Hence, sphingosine and sphingosine kinase were suggested to be involved. From this theory, several studies carried out in various models have shown that isoflurane provides neuroprotection by activating this pathway, which then upregulates the ERK/ MAPK/Akt pathway, resulting in increased cell growth and survival. However, newer theories indicate a more protein-based mechanism of action, via several ion channels, as well as GABA and glutamate receptors. The involvement of iNOS in isoflurane-mediated neuroprotection is another popular mechanism proposed and is more in line with the Protein Theory. Notably, many of these pathways, including sphingosine-1-phosphate and iNOS, exhibit protection via the activation of the ERK/MAPK/Akt pathway. This is significant and may be the way to connect the different pathways implicated, thereby establishing how isoflurane is protecting neurons from cell death.

Finally, neonatal HI results from several different patholophysiological manifestations. Hence, it may be necessary to target more than one of these to provide sufficient neuroprotection. Consequently, using isoflurane along with another intervention may be much more effective. Moreover, with the suggestion by some studies that isoflurane is merely delaying repercussions from ischemia, rather than preventing them, this may open a longer therapeutic window for other interventions to be applied, and may also provide an additive protective effect.

The possibility of using isoflurane clinically is very promising, and with the fact that it is already so widely used, addressing the current concerns would prove very beneficial in progressing not only the field of neonatal brain injury, but also many diseases related to hypoxia and ischemia.

\section{Abbreviations}

MAC minimum alveolar concentration

LPS Lipopolysaccharide

SAH Subarachnoid hemorrhage

MCAO Middle Cerebral Artery Occlusion

HI Hypoxia Ischemia

GABA $\quad \mathrm{Y}$-aminobutyric acid

PI3K Phosphoinositol-3-Kinase

AKT Protein Kinase B

MAPK Mitogen-activated Protein Kinase

ERK Extracellular Signal-Related Kinase

Bcl-2 B-cell lymphoma 2

iNOS inducible Nitric Oxide Synthase

S1P Sphingosine-1-phosphate

SK Sphingosine kinase

LDH lactate dehydrogenase

CK-MB Creatine kinase MB 
PKCe Protein Kinase $\mathrm{C} \epsilon$

ALDH2 Aldehyde dehydrogenase 2

BBB Blood brain barrier

NF-kB Nuclear Factor- KB

\section{References}

1. Matchett GA, Allard MW, Martin RD, Zhang JH. Neuroprotective effect of volatile anesthetic agents: molecular mechanisms. Neurol Res. 2009 Mar; 31(2):128-134. [PubMed: 19298752]

2. Loepke AW, McCann JC, Kurth CD, McAuliffe JJ. The physiologic effects of isoflurane anesthesia in neonatal mice. Anesth Analg. 2006 Jan; 102(1):75-80. [PubMed: 16368807]

3. Antkowiak B. How do general anaesthetics work? Naturwissenschaften. 2001; 88(5):201-213. [PubMed: 11482433]

4. Zuo Z. Are volatile anesthetics neuroprotective or neurotoxic? Med Gas Res. 2012; 2(1):10. [PubMed: 22510328]

5. Eger EI 2nd. Isoflurane: a review. Anesthesiology. 1981 Nov; 55(5):559-576. [PubMed: 7027831]

6. Eger EI 2nd. The pharmacology of isoflurane. Br J Anaesth. 1984; 56(Suppl 1):71S-99S. [PubMed: 6391530]

7. Chung IS, Kim JA, Kim JA, et al. Reactive oxygen species by isoflurane mediates inhibition of nuclear factor kappaB activation in lipopolysaccharide-induced acute inflammation of the lung. Anesth Analg. 2013 Feb; 116(2):327-335. [PubMed: 23302986]

8. Harr JN, Moore EE, Stringham J, et al. Isoflurane prevents acute lung injury through ADP-mediated platelet inhibition. Surgery. 2012 Aug; 152(2):270-276. [PubMed: 22828148]

9. Kinoshita H, Matsuda N, Iranami H, et al. Isoflurane pretreatment preserves adenosine triphosphatesensitive $\mathrm{K}(+)$ channel function in the human artery exposed to oxidative stress caused by high glucose levels. Anesth Analg. 2012 Jul; 115(1):54-61. [PubMed: 22467893]

10. Kim M, Kim M, Kim N, D'Agati VD, Emala CW Sr, Lee HT. Isoflurane mediates protection from renal ischemia-reperfusion injury via sphingosine kinase and sphingosine-1-phosphate-dependent pathways. Am J Physiol Renal Physiol. 2007 Dec; 293(6):F1827-F1835. [PubMed: 17898040]

11. Lee HT, Ota-Setlik A, Fu Y, Nasr SH, Emala CW. Differential protective effects of volatile anesthetics against renal ischemia-reperfusion injury in vivo. Anesthesiology. $2004 \mathrm{Dec} ; 101(6)$ : 1313-1324. [PubMed: 15564938]

12. Lang XE, Wang X, Zhang KR, Lv JY, Jin JH, Li QS. Isoflurane preconditioning confers cardioprotection by activation of ALDH2. PLoS One. 2013; 8(2):e52469. [PubMed: 23468836]

13. Altay O, Suzuki H, Hasegawa Y, et al. Isoflurane attenuates blood-brain barrier disruption in ipsilateral hemisphere after subarachnoid hemorrhage in mice. Stroke. 2012 Sep; 43(9):25132516. [PubMed: 22773559]

14. Li H, Yin J, Li L, Deng J, Feng C, Zuo Z. Isoflurane postconditioning reduces ischemia-induced nuclear factor-kappaB activation and interleukin 1beta production to provide neuroprotection in rats and mice. Neurobiol Dis. 2013 Jun.54:216-224. [PubMed: 23313315]

15. Li L, Zuo Z. Isoflurane postconditioning induces neuroprotection via Akt activation and attenuation of increased mitochondrial membrane permeability. Neuroscience. $2011 \mathrm{Dec}$ 29.199:44-50. [PubMed: 22040798]

16. Khatibi NH, Ma Q, Rolland W, et al. Isoflurane posttreatment reduces brain injury after an intracerebral hemorrhagic stroke in mice. Anesth Analg. 2011 Aug; 113(2):343-348. [PubMed: 21596881]

17. Statler KD, Alexander H, Vagni V, et al. Isoflurane exerts neuroprotective actions at or near the time of severe traumatic brain injury. Brain Res. 2006 Mar 3; 1076(1):216-224. [PubMed: 16473332] 
18. Zhou Y, Lekic T, Fathali N, et al. Isoflurane posttreatment reduces neonatal hypoxic-ischemic brain injury in rats by the sphingosine-1-phosphate/phosphatidylinositol-3-kinase/Akt pathway. Stroke. $2010 \mathrm{Jul}$; 41(7):1521-1527. [PubMed: 20508187]

19. Vannucci SJ, Hagberg H. Hypoxia-ischemia in the immature brain. J Exp Biol. 2004 Aug; 207(Pt 18):3149-3154. [PubMed: 15299036]

20. Sasaoka N, Kawaguchi M, Kawaraguchi Y, et al. Isoflurane exerts a short-term but not a long-term preconditioning effect in neonatal rats exposed to a hypoxic-ischaemic neuronal injury. Acta Anaesthesiol Scand. 2009 Jan; 53(1):46-54. [PubMed: 19032558]

21. Ferriero DM. Neonatal brain injury. N Engl J Med. 2004 Nov 4; 351(19):1985-1995. [PubMed: 15525724]

22. Zhao P, Zuo ZY. Isoflurane preconditioning induces neuroprotection that is inducible nitric oxide synthase-dependent in neonatal rats. Anesthesiology. 2004 Sep; 101(3):695-702. [PubMed: 15329594]

23. RJ, V RC, B JB. The influence of immaturity on hypoxic-ischemic brain damage in the rat. Ann Neurol. 1981; 9(2):131-141. [PubMed: 7235629]

24. Vannucci RC, Connor JR, Mauger DT, et al. Rat model of perinatal hypoxic-ischemic brain damage. J Neurosci Res. 1999 Jan 15; 55(2):158-163. [PubMed: 9972818]

25. Chen H, Burris M, Fajilan A, Spagnoli F, Tang J, Zhang JH. Prolonged Exposure to Isoflurane Ameliorates Infarction Severity in the Rat Pup Model of Neonatal Hypoxia-Ischemia. Transl Stroke Res. 2011 Sep 1; 2(3):382-390. [PubMed: 21892364]

26. Warner DS. Isoflurane neuroprotection: a passing fantasy, again? Anesthesiology. 2000; 92(5)

27. Bickler PE, Fahlman CS. The inhaled anesthetic, isoflurane, enhances Ca2+-dependent survival signaling in cortical neurons and modulates MAP kinases, apoptosis proteins and transcription factors during hypoxia. Anesth Analg. 2006 Aug; 103(2):419-429. table of contents. [PubMed: 16861427]

28. Gray JJ, Bickler PE, Fahlman CS, Zhan X, Schuyler JA. Isoflurane neuroprotection in hypoxic hippocampal slice cultures involves increases in intracellular $\mathrm{Ca} 2+$ and mitogen-activated protein kinases. Anesthesiology. 2005 Mar; 102(3):606-615. [PubMed: 15731600]

29. Jamnicki-Abegg M, Weihrauch D, Pagel PS, et al. Isoflurane inhibits cardiac myocyte apoptosis during oxidative and inflammatory stress by activating Akt and enhancing Bcl-2 expression. Anesthesiology. 2005 Nov; 103(5):1006-1014. [PubMed: 16249675]

30. Gwak MS, Cao L, Li L, Zuo Z. Isoflurane preconditioning reduces oxygen-glucose deprivationinduced neuronal injury via B-cell lymphoma 2 protein. Environ Toxicol Pharmacol. 2011 Jan; 31(1):262-265. [PubMed: 21359097]

31. Li QF, Zhu YS, Jiang H. Isoflurane preconditioning activates HIF-1alpha, iNOS and Erk1/2 and protects against oxygen-glucose deprivation neuronal injury. Brain Res. 2008 Dec 15.1245:26-35. [PubMed: 18930717]

32. Theilmeier G, Schmidt C, Herrmann J, et al. High-density lipoproteins and their constituent, sphingosine-1-phosphate, directly protect the heart against ischemia/reperfusion injury in vivo via the S1P3 lysophospholipid receptor. Circulation. 2006 Sep 26; 114(13):1403-1409. [PubMed: 16982942]

33. Man K, Ng KT, Lee TK, et al. FTY720 attenuates hepatic ischemia-reperfusion injury in normal and cirrhotic livers. Am J Transplant. 2005 Jan; 5(1):40-49. [PubMed: 15636610]

34. Lochhead KM, Zager RA. Fluorinated anesthetic exposure "activates" the renal cortical sphingomyelinase cascade. Kidney Int. 1998 Aug; 54(2):373-381. [PubMed: 9690203]

35. Pyne S, Pyne N. Sphingosine 1-phosphate signalling and termination at lipid phosphate receptors. Biochimica et Biophysica Acta (BBA) - Molecular and Cell Biology of Lipids. 2002; 1582(1-3): 121-131.

36. Bakar AM, Park SW, Kim M, Lee HT. Isoflurane Protects Against Human Endothelial Cell Apoptosis by Inducing Sphingosine Kinase-1 via ERK MAPK. Int J Mol Sci. 2012; 13(1):977993. [PubMed: 22312298]

37. Pitson SM, Moretti PA, Zebol JR, et al. Activation of sphingosine kinase 1 by ERK1/2-mediated phosphorylation. EMBO J. 2003 Oct 15; 22(20):5491-5500. [PubMed: 14532121] 
38. Kim M, Park SW, Kim M, D'Agati VD, Lee HT. Isoflurane activates intestinal sphingosine kinase to protect against renal ischemia-reperfusion-induced liver and intestine injury. Anesthesiology. 2011 Feb; 114(2):363-373. [PubMed: 21245730]

39. Franks NP, Lieb WR. Where do general anaesthetics act? Nature. 1978 Jul 27; 274(5669):339_ 342. [PubMed: 672957]

40. Zhao P, Peng L, Li L, Xu X, Zuo Z. Isoflurane preconditioning improves long-term neurologic outcome after hypoxic-ischemic brain injury in neonatal rats. Anesthesiology. $2007 \mathrm{Dec} ; 107(6)$ : 963-970. [PubMed: 18043065]

41. Zuo Z, Wang Y, Huang Y. Isoflurane preconditioning protects human neuroblastoma SH-SY5Y cells against in vitro simulated ischemia-reperfusion through the activation of extracellular signalregulated kinases pathway. Eur J Pharmacol. 2006 Aug 7; 542(1-3):84-91. [PubMed: 16806162]

42. Zheng S, Zuo Z. Isoflurane preconditioning induces neuroprotection against ischemia via activation of P38 mitogen-activated protein kinases. Mol Pharmacol. 2004 May; 65(5):1172-1180. [PubMed: 15102945]

43. Kapinya KJ, Lowl D, Futterer C, et al. Tolerance against ischemic neuronal injury can be induced by volatile anesthetics and is inducible NO synthase dependent. Stroke. 2002 Jul; 33(7):18891898. [PubMed: 12105371]

44. Huang PL. Nitric oxide and cerebral ischemic preconditioning. Cell Calcium. 2004 Sep-Oct;36(34):323-329. [PubMed: 15261488]

45. McAuliffe JJ, Joseph B, Vorhees CV. Isoflurane-delayed preconditioning reduces immediate mortality and improves striatal function in adult mice after neonatal hypoxia-ischemia. Anesth Analg. 2007 May; 104(5):1066-1077. tables of contents. [PubMed: 17456654]

46. Kawaguchi M, Kimbro JR, Drummond JC, Cole DJ, Kelly PJ, Patel PM. Isoflurane Delays but Does Not Prevent Cerebral Infarction in Rats Subjected to Focal Ischemia. Anesthesiology. 2000; 92(5):1226-1228. [PubMed: 10781264]

47. McAuliffe JJ, Loepke AW, Miles L, Joseph B, Hughes E, Vorhees CV. Desflurane, isoflurane, and sevoflurane provide limited neuroprotection against neonatal hypoxia-ischemia in a delayed preconditioning paradigm. Anesthesiology. 2009 Sep; 111(3):533-546. [PubMed: 19672176]

48. Jevtovic-Todorovic V, Hartman RE, Izumi Y, et al. Early exposure to common anesthetic agents causes widespread neurodegeneration in the developing rat brain and persistent learning deficits. Journal of Neuroscience. 2003 Feb 1; 23(3):876-882. [PubMed: 12574416] 


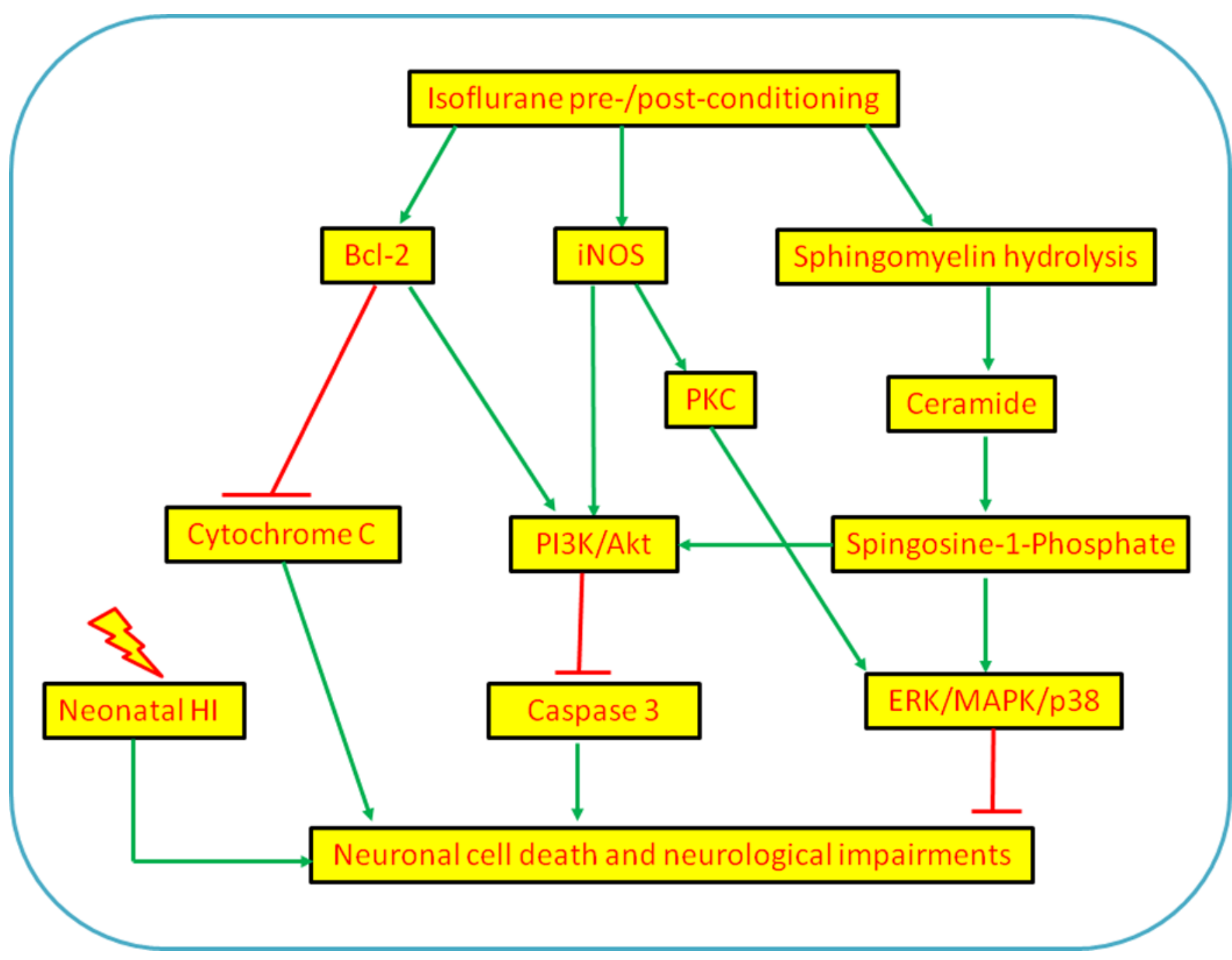

Figure 1.

Proposed Mechanisms of Isoflurane Neuroprotection. Other potential mechanisms have been explored; however, these are the most extensively addressed in this review ${ }^{18,29,40,41}$. 


\section{Table 1}

Isoflurane shows protection in various animal models

\begin{tabular}{|c|c|c|}
\hline Animal Model & Isoflurane Protection & Source \\
\hline \multicolumn{3}{|c|}{ General Models } \\
\hline $\begin{array}{l}\text { LPS-Induced Actute Lung Inflammation } \\
\text { (Rat) }\end{array}$ & $\begin{array}{l}\text { Inhibited ROS burst, NF-KB activation, and pro-inflammatory } \\
\text { cytokines }\end{array}$ & Chung, I.S. et.al. 2013 \\
\hline T/HS-induced ALI & Inhibited platelets through the ADP pathway & Harr, N. et.al. 2012 \\
\hline Renal Ischemia/ Reperfusion Injury & $\begin{array}{l}\text { Reduced renal dysfunction and tubular necrosis via the SK/S1P } \\
\text { pathway }\end{array}$ & $\begin{array}{l}\text { Kim, M. et.al.2007; Lee, } \\
\text { H.T. et.al. } 2004\end{array}$ \\
\hline Cardiac Injury & $\begin{array}{l}\text { Attenuated the release of } \mathrm{LDH} \text { and } \mathrm{CK}-\mathrm{MB} \text {, and reduced infarct } \\
\text { size via } \mathrm{PKC} \in \mathrm{ALDH} 2 \text { pathway }\end{array}$ & Lang, X. et.al. 2013 \\
\hline $\begin{array}{l}\text { High Glucose-induced Oxidative Stress } \\
\text { in Isolated Human Artery }\end{array}$ & $\begin{array}{l}\text { Preserved vascular function by restoring ATP-sensitive } \mathrm{K}^{+} \\
\text {channel function }\end{array}$ & Kinoshita, H. et.al. 2012 \\
\hline \multicolumn{3}{|c|}{ Stroke Models } \\
\hline Subarachnoid Hemorrhage & $\begin{array}{l}\text { Prevented post SAH neuronal apoptosis and BBB disruption in } \\
\text { ipsilateral hemisphere through S1P pathway }\end{array}$ & Altay, O. et.al. 2012 \\
\hline Middle Cerebral Artery Occlusion & $\begin{array}{l}\text { Improved neurological outcome, and reduced inflammatory } \\
\text { cytokine production via attenuation of NF- } \mathrm{kB} \text { activation }\end{array}$ & Li, H. et.al. 2013 \\
\hline Intracerebral Hemorrhage & $\begin{array}{l}\text { Reduced brain edema, apoptotic cell death, and neurological } \\
\text { deficits }\end{array}$ & Khatibi, N. H. et.al. 2011 \\
\hline Traumatic Brain Injury & Increased CA3 neuronal survival, and improved histopathology & Statler, K.D. et.al. 2006 \\
\hline Neonatal Hypoxic Ischemic Brain Injury & $\begin{array}{l}\text { Reduced infarction volume, improved neurobehavioral outcome, } \\
\text { and increased pAkt }\end{array}$ & Zhou, Y. et.al. 2010 \\
\hline
\end{tabular}

